\title{
Neurokinin-1 Receptor Antagonism Ameliorates Dry Eye Disease by Inhibiting Antigen-Presenting Cell Maturation and T Helper 17 Cell Activation
}

\author{
Man Yu, ${ }^{* \dagger \dagger}$ Sang-Mok Lee, ${ }^{* \dagger}$ Hyunsoo Lee, ${ }^{* \dagger}$ Afsaneh Amouzegar, ${ }^{* \dagger}$ Takeshi Nakao, ${ }^{* \dagger}$ Yihe Chen, ${ }^{* \dagger}$ and Reza Dana ${ }^{* \dagger}$
}

From the Schepens Eye Research Institute, ${ }^{*}$ Massachusetts Eye and Ear Infirmary, and the Department of Ophthalmology, ${ }^{\dagger}$ Harvard Medical School, Boston, Massachusetts; and the Department of Ophthalmology, ${ }^{\ddagger}$ Sichuan Academy of Medical Sciences and Sichuan Provincial People’s Hospital, Chengdu, China

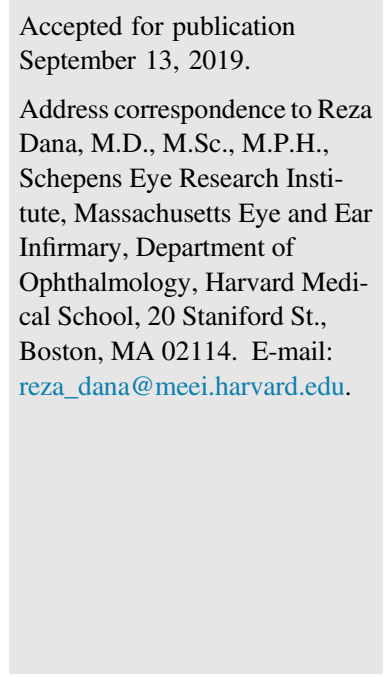

Accepted for publication September 13, 2019.

Dana, M.D., M.Sc., M.P.H., Schepens Eye Research Institute, Massachusetts Eye and Ear Infirmary, Department of Ophthalmology, Harvard Medical School, 20 Staniford St. Boston, MA 02114. E-mail: reza_dana@meei.harvard.edu.

\begin{abstract}
Neuroinflammation plays an important role in the pathogenesis of ocular surface disease, including dry eye disease (DED), but little is known about the contribution of substance $P(S P)$ to DED. In this study, we investigated the expression of SP at the ocular surface and evaluated its effect on maturation of antigen-presenting cells (APCs), the key cell component involved in the induction of type 17 helper Tcell (Th17) response in DED. The effect of topical blockade of SP signaling was further investigated using neurokinin-1 receptor (NK1R) inhibitors on APC maturation, Th17 cell activation, and disease severity in a mouse model of DED. The results demonstrate that SP is constitutively expressed at the ocular surface, and trigeminal ganglion neurons are the major source of SP in DED. SP derived from trigeminal ganglion enhanced the expression of major histocompatibility complex class II maturation marker by bone marrow-derived dendritic cells, an effect that is abrogated by blockade of SP signaling using NK1R antagonist spantide. Finally, using a well-established murine model of DED, topical treatment of DED mice with NK1R antagonists CP-99,994 and L-733,060 suppressed APC acquisition of major histocompatibility complex class II, reduced Th17 cell activity, and ameliorated DED severity. These findings are of translational value, as they suggest that antagonizing NK1R-mediated SP signaling may be an effective strategy in suppressing Th17-mediated ocular surface disease. (Am J Pathol 2020, 190: 125-133; https://doi.org/10.1016/j.ajpath.2019.09.020)
\end{abstract}

Dry eye disease (DED) is a highly prevalent ocular disorder, which causes ocular discomfort and visual disturbance that affects millions of individuals worldwide. ${ }^{1,2}$ Although several factors play key roles in development of DED, the prominent role of inflammation in the disease pathogenesis has been increasingly recognized. ${ }^{3}$ Several immunomodulatory medications, such as topical applications of cyclosporine and lifitegrast, have been approved for treatments for moderate-to-severe $\mathrm{DED}^{4}$; however, drug adverse effects limit their application in many patients.

Activation of antigen-presenting cells (APCs) is a key step in the pathogenesis of DED. Mature APCs migrate from the inflamed cornea to draining lymph nodes (DLNs) via afferent lymphatics, where they prime naïve $\mathrm{T}$ cells to expand and differentiate into $\mathrm{CD}^{+}$type 1 helper $\mathrm{T}$ cell
(Th1) and Th17 effectors., ${ }^{5,6}$ Th17 cells are the principal effector cell component in DED; these cells mediate the disruption of corneal barrier on infiltration to the cornea. This concept is supported by several studies, which demonstrate increased frequencies of Th17 cells and overexpression of Th17-associated cytokines in mice with $\mathrm{DED}^{6,7}$ and the efficacy of in vivo blockade of IL-17 in suppressing ocular surface disease. ${ }^{3}$

Substance P (SP), an 11-amino acid neuropeptide of the tachykinin family, is derived from preprotachykinin A

\footnotetext{
Supported by NIH/National Eye Institute grant R01 EY 20889 (R.D.) and core grant P30EY003790.

M.Y. and S.-M.L. contributed equally to this work.

Disclosures: None declared.
} 
protein and is expressed by diverse cell types. ${ }^{8-10}$ SP has long been recognized as an active mediator of inflammation $^{11}$ and wound healing. ${ }^{12-14}$ These processes are mediated through SP's interaction with the neurokinin-1 receptor (NK1R), a member of the neurokinin family of G-protein-coupled receptors. ${ }^{15}$ Increased SP levels have been reported in the cornea of mice after alkali burn, ${ }^{16}$ and elevated tear SP levels are observed up to 3 months after LASIK (laser-assisted in situ keratomileusis) and are associated with reduced nerve fiber density and subsequent development of dry eye symptoms. ${ }^{17}$ The efficacy of NK1R antagonists has been tested in several corneal diseases, including pseudomonas keratitis, ${ }^{18}$ herpetic stromal keratitis, ${ }^{19}$ and corneal neovascularization. ${ }^{20}$ The results of these studies have shown that inhibition of SP signaling, using NK1R antagonists, reduces the inflammation associated with microbial keratitis and suppresses corneal neovascularization. ${ }^{18-20}$ SP has been shown to amplify the inflammatory response in the central nervous system through its ability to skew the inflammatory response toward Th17 immunity. ${ }^{21}$ In addition, SP as a key player in cellular migration can regulate dendritic cell (DC) homing to draining lymph nodes through modulating the surface expression of chemokine receptors and adhesion molecules by these cells. ${ }^{22,23}$

Despite the implied role of neuroinflammation in the chronicity of epitheliopathy in DED, the precise role of SP in the pathogenesis of DED, in particular the effect of blocking SP signaling on the severity of immune response and epitheliopathy in DED, has not yet been elucidated. Herein, we evaluated DED-induced alterations in SP expression and investigated the effect of SP derived from stimulated corneal nerve endings on APC maturation, a key step in activation of effector Th17 mechanisms in DED. Furthermore, we evaluated the efficacy of blocking SP signaling using NK1R antagonists in reducing DED severity. Our results show that SP is constitutively expressed at the ocular surface, and its expression is up-regulated in the course of DED. Using in vitro studies, we demonstrate that SP augments the maturation of bone marrow-derived dendritic cells, and antagonizing NK1R abrogates this effect. Finally, using a well-established mouse model of DED, we show that treatment of DED mice with topical NK1R antagonists CP-99,994 and L-733,060 suppresses APC maturation and Th17 cell activation and significantly reduces disease severity.

\section{Materials and Methods}

\section{Animals}

Female C57BL/6 mice, aged 8 to 9 weeks (Charles River Laboratories, Wilmington, MA), were used in these experiments. All animal experiments were approved by the Schepens Eye Research Institute Animal Care and Use
Committee and adhered to the Association for Research in Vision and Ophthalmology Statement for the Use of Animals in Ophthalmic and Vision Research.

\section{Induction of DED}

DED was induced by housing the mice in a low-humidity (relative humidity, $<20 \%$ ) controlled environment chamber with constant airflow of $15 \mathrm{~L} /$ minute and temperature of $21^{\circ} \mathrm{C}$ to $23^{\circ} \mathrm{C}$ for 14 days. Age- and sex-matched mice housed in room air conditions served as controls. ${ }^{24}$ Corneal epithelial disease was evaluated using fluorescein (Sigma-Aldrich, St. Louis, MO) staining and scored using the National Eye Institute (Bethesda, MD) grading system. ${ }^{25}$ Briefly, $1 \mu \mathrm{L}$ of $2.5 \%$ fluorescein was applied into the lateral conjunctival sac of the mice; and after 3 minutes, corneas were examined with a slit-lamp biomicroscope under cobalt blue light. Punctate staining was recorded in a masked manner with the standard National Eye Institute grading system of 0 to 3 for each of the five areas of the cornea.

\section{Topical Treatment with NK1R Antagonist}

Mice were assigned to one of the four groups ( $n=5$ each). A total of $1 \mu \mathrm{g} / \mu \mathrm{L}$ of NK1R antagonist CP-99,994 or L-733,060 (R\&D Systems, Minneapolis, MN) or phosphatebuffered saline (PBS) was administered topically three times per day from day 4 to day 14 after DED induction. Untreated DED mice served as controls.

\section{Generation of BMDCs}

Long bones (femur and tibia) were harvested from C57BL/6 mice, and cell suspension was prepared. Cells were incubated with red blood cell lysis buffer (Sigma-Aldrich) at $37^{\circ} \mathrm{C}$ for 10 minutes. Bone marrow cells were plated at the concentration of $5 \times 10^{6}$ cells in 5 mL RPMI 1640 medium/ well (Lonza Biologics, Inc., Hopkinton, MA) supplemented with 5\% heat-inactivated fetal calf serum (Atlanta Biologicals, Flower Branch, GA), $2 \mathrm{mmol} / \mathrm{L}$ L-glutamine (Lonza Biologics, Inc.), $100 \mathrm{U} / \mathrm{mL}$ of penicillin (Lonza Biologics, Inc.), $100 \mu \mathrm{g} / \mathrm{mL}$ of streptomycin (Lonza Biologics, Inc.), $50 \mathrm{mmol} / \mathrm{L}$ 2-mercaptoethanol (SigmaAldrich), and $20 \mathrm{ng} / \mathrm{mL}$ of granulocyte/macrophage colonystimulating factor (BioLegend, San Diego, CA) for 6 to 7 days. Lymphocytes were removed by washing on days 2 and 4 of culture. On day 7, nonadherent and loosely adherent immature bone marrow-derived dendritic cells (BMDCs) were harvested. To activate BMDCs, immature BMDCs were cultured in the presence of $20 \mathrm{ng} / \mathrm{mL}$ of IL-1 $\beta$ (PeproTech, Rocky Hill, NJ) in 6-well plates for 24 hours.

\section{Primary TG Culture}

Trigeminal ganglions (TGs) were primarily cultured, as described before. ${ }^{26}$ Briefly, TGs were harvested from 6- to 
8-week-old C57BL/6 mice and were digested with papain, followed by collagenase type II/dispase (Invitrogen, Carlsbad, CA). The TGs were selected by the lower layers of the five-layered OptiPrep density gradients (Sigma-Aldrich). Neurons were counted and plated on poly-L-lysine and laminin-coated four-chamber slides or 24-well plates at a density of 3000 cells per well. ${ }^{27}$ Neuronal cultures were maintained with complete neuronal medium, consisting of Neurobasal A medium (Invitrogen; catalog number 10888022) supplemented with $2 \%$ B27 supplement; $1 \%$ penicillin and streptomycin; $500 \mu \mathrm{mol} / \mathrm{L}$ L-glutamine; $50 \mathrm{ng} / \mathrm{mL}$ nerve growth factor; $50 \mathrm{ng} / \mathrm{mL}$ glial cell-derived neurotrophic factor; and the mitotic inhibitors fluorodeoxyuridine $(40 \mu \mathrm{mol} / \mathrm{L})$ and aphidicolin $(16.6 \mu \mathrm{g} / \mathrm{mL})$ for the first 3 days. The medium was then replaced with fresh medium without fluorodeoxyuridine and aphidicolin (growth factors were from R\&D Systems, and other supplements were from Sigma-Aldrich).

\section{Bone Marrow-Derived Dendritic Cell and Trigeminal Ganglion Co-Culture}

Primary TG culture was performed, and loosely adherent immature BMDCs were collected after 7 days of culture. After counting the number of BMDCs, the cell density was adjusted to 150,000 cells $/ \mathrm{mL}$ in BMDC culture media (based on RPMI 1640 medium) without granulocyte/macrophage colony-stimulating factor. After removal of the TG culture medium, $1 \mathrm{~mL}$ of media containing immature BMDCs was added to each well. After 2 hours, IL-1 $\beta(20 \mathrm{ng} / 10 \mu \mathrm{L})$ was added to each well to induce BMDC maturation, and cells were co-cultured for 18 hours.

\section{Single-Cell Suspension Preparation and Flow Cytometry}

Submandibular and cervical DLNs were harvested, and single-cell suspensions were prepared. Cells were stimulated with $50 \mathrm{ng} / \mathrm{mL}$ phorbol 12-myristate 13-acetate (SigmaAldrich) and $500 \mathrm{ng} / \mathrm{mL}$ ionomycin (Sigma-Aldrich) for 6 hours in the presence of a commercial protein transport inhibitor $(0.7 \mu \mathrm{L} / 100 \mu \mathrm{L}$ media; Golgistop; BD Biosciences, San Jose, CA). Conjunctivae were harvested by lifting at the junction of bulbar and palpebral conjunctiva and dissecting along both bulbar and palpebral insertion points (Vannas Scissors; Storz, Bausch \& Lomb, Rancho Cucamonga, CA). Conjunctiva samples were cultured in RPMI 1640 medium (Thermo Fisher Scientific, Waltham, MA) supplemented with $10 \%$ fetal bovine serum and stimulated with $50 \mathrm{ng} / \mathrm{mL}$ phorbol 12-myristate 13-acetate (Sigma-Aldrich) and $500 \mathrm{ng}$ / $\mathrm{mL}$ ionomycin (Sigma-Aldrich) in the presence of a protein transport inhibitor $\left(0.7 \mu \mathrm{L} / 100 \mu \mathrm{L}\right.$ media; Golgistop) at $37^{\circ} \mathrm{C}$ for 24 hours. Harvested corneas were digested in RPMI 1640 media containing $2 \mathrm{mg} / \mathrm{mL}$ collagenase type IV (SigmaAldrich) and $2 \mathrm{mg} / \mathrm{mL}$ DNase I (Roche, Indianapolis, IN) for 1 hour at $37^{\circ} \mathrm{C}$. The suspension was then filtered through a $70-\mu \mathrm{m}$ cell strainer. Single-cell suspensions of DLN and cornea were stained with the following antibodies: Brilliant Violet 421-conjugated anti-mouse I-A/I-E [major histocompatibility complex class II (MHC-II)] (BioLegend), phosphatidylethanolamine-conjugated anti-CD11c (BD Pharmingen, San Jose, CA), PerCP/Cy5.5-conjugated antiCD11b, fluorescein isothiocyanate-conjugated anti-CD4 (BioLegend), and phosphatidylethanolamine-conjugated anti-IL-17A (eBioscience, San Diego, CA). Intranuclear staining with phosphatidylethanolamine/Cy7-conjugated antiFoxp3 (eBioscience) was performed after fixation and permeabilization of cells. Control samples were stained with appropriate isotype-matched antibodies. Stained cells were examined using an LSRII Flow Cytometer (BD Biosciences, Franklin Lakes, NJ), and data were analyzed using commercial Summit software version 4.3 (Dako Colorado, Inc., Fort Collins, CO).

\section{Enzyme-Linked Immunosorbent Assay}

Corneas and TGs were harvested on days 4 and 14 after DED induction, and protein levels of SP in both tissues were evaluated using enzyme-linked immunosorbent assay. For protein extraction, harvested cornea and TGs were stored in cold sterile PBS containing protease inhibitors (SigmaAldrich) at $-80^{\circ} \mathrm{C}$ until used. The samples were homogenized on ice and centrifuged. The supernatant was assayed for levels of SP using a commercial competitive enzyme immunoassay kit (R\&D Systems).

\section{Real-Time PCR}

Bulbar and palpebral conjunctivae, corneas, and TGs were harvested after DED induction, and mRNA levels of SP in both tissues were evaluated using real-time PCR. Harvested tissues were stored in TRIzol reagent (Invitrogen) at $-80^{\circ} \mathrm{C}$ until RNA was isolated and reverse transcribed using RNeasy micro kit (Qiagen, Valencia, CA) and SuperScript III kit (Invitrogen). Real-time PCR was performed using TaqMan Universal PCR Master Mix and predesigned primers for IL-17A (Mm00439618_m1), SP (Mm01166996_m1), and glyceraldehyde 3-phosphate dehydrogenase (Mm99999915_g1; Applied Biosystems, Carlsbad, CA). Samples were analyzed using a real-time PCR system (LightCycler 480 II System; Roche Applied Science, Indianapolis, IN), and results were analyzed by the comparative $\mathrm{C}_{\mathrm{T}}$ method, using glyceraldehyde 3 -phosphate dehydrogenase as an internal control.

\section{Statistical Analysis}

Unpaired $t$-test was performed for comparison between two groups, and $P<0.05$ was considered statistically significant. Results are presented as means \pm SEM of at least two independent experiments. 


\section{Results}

\section{Substance P Expression Increases in the Course of Dry Eye Disease}

Levels of expression of SP in different phases of DED were evaluated by measuring SP mRNA and protein levels in the cornea, conjunctiva, and TG of normal and DED mice. SP protein levels were assessed in the TG culture supernatant using enzyme-linked immunosorbent assay. Our results demonstrated baseline expression of SP in both the ocular surface (cornea and conjunctiva) and TG, levels of which were up-regulated after induction of DED (Figure 1A). At the mRNA level, expression of SP in corneas and conjunctiva of DED mice was significantly higher than healthy controls [1.84- and 12.09-fold on day $4(P=0.001$ and $P=0.01$, respectively); and 1.43- and 12.34-fold on day 14 $(P=0.02$ and $P=0.04$, respectively)] (Figure 1B). Furthermore, in the TG, SP mRNA levels were significantly higher than those seen in the cornea or conjunctiva, and increased expression induced by desiccating stress occurred later in the course of DED. These results show constitutive expression of SP at the ocular surface mainly in the TG neurons, levels of which are up-regulated in response to desiccating stress.
SP Derived from Trigeminal Nerves Promotes Bone Marrow-Derived Dendritic Cell Maturation in Vitro

Mature APCs play a key role in the activation of Th17 cells and the autoimmune response in DED. ${ }^{28-30}$ Given the observation of increased SP levels in response to desiccating stress, the effect of SP was studied on the maturation of APCs. Primed BMDCs were cultured in the presence of three different concentrations of SP $(25,50$, and $100 \mathrm{pg} / \mathrm{mL})$ for 18 hours, and expression of MHC-II maturation marker by BMDC was evaluated using flow cytometry. Increasing concentrations of SP led to a significant and dose-dependent increase in the mean fluorescence intensity of MHC-II in CD11 $\mathrm{c}^{+}$BMDCs (Figure 2A). To investigate the potential role of TG neurons (which innervate the cornea) on BMDC maturation, TGs harvested from DED or control mice were cultured for 3 days and subsequently co-cultured with primed BMDCs for 18 hours. In addition, two different doses of neurokinin-1 receptor antagonist, spantide (10 and $100 \mu \mathrm{mol} / \mathrm{L}$ ), were added to the co-culture system to block the SP signaling. The expression of MHC-II maturation marker by $\mathrm{CD}_{11 \mathrm{c}^{+}}$BMDCs was analyzed in different groups using flow cytometry. TG derived from DED mice induced a significant increase in expression of MHC-II by BMDC compared with TG derived from normal healthy
A
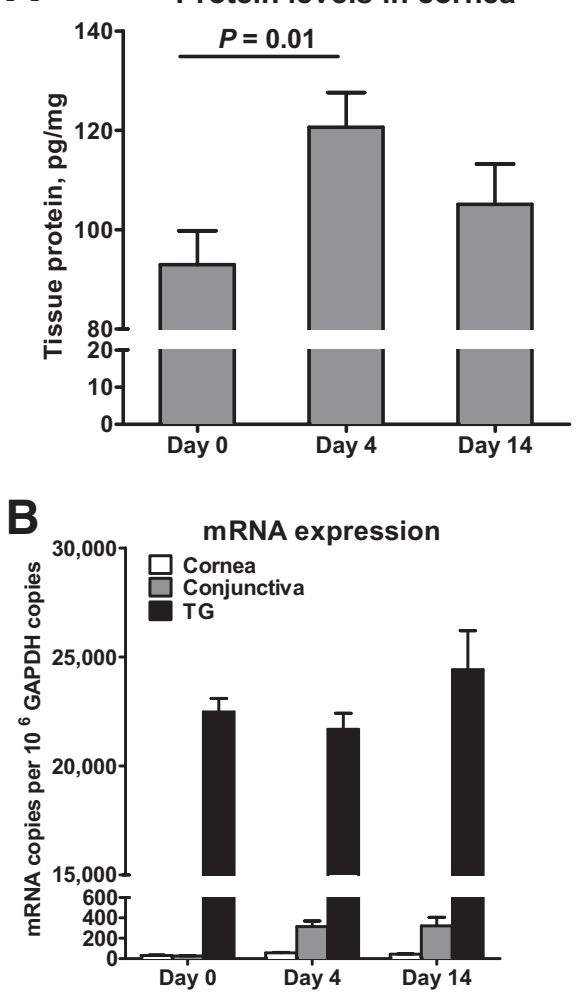
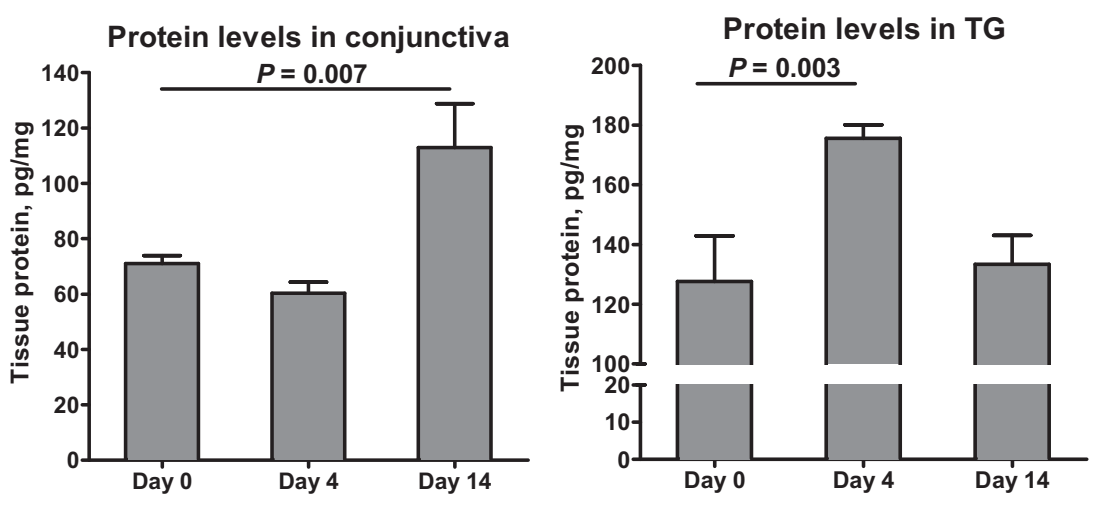

Figure 1 Substance $P$ levels increase in the ocular surface in the course of dry eye disease (DED). DED was induced in C57BL/6 mice for 14 days. Corneas, conjunctiva, and trigeminal ganglia (TGs) of control (day 0) and DED mice (days 4 and 14) were harvested; and tissues were homogenized. A: Protein levels of substance $P$ in the cornea, conjunctiva, and TG were analyzed in the supernatant of homogenized tissues of control and DED mice using enzyme-linked immunosorbent assay; and protein levels were adjusted to the total protein measured by the BCA Protein assay kit (Thermo Scientific, Rockford, IL). B: Substance P mRNA levels in the cornea, conjunctiva, and TG of control and DED mice were analyzed using real-time PCR. Data are expressed as means \pm SEM (A and $\mathbf{B}) . n=2$ independent experiments ( $\mathbf{A}$ and $\mathbf{B})$. 


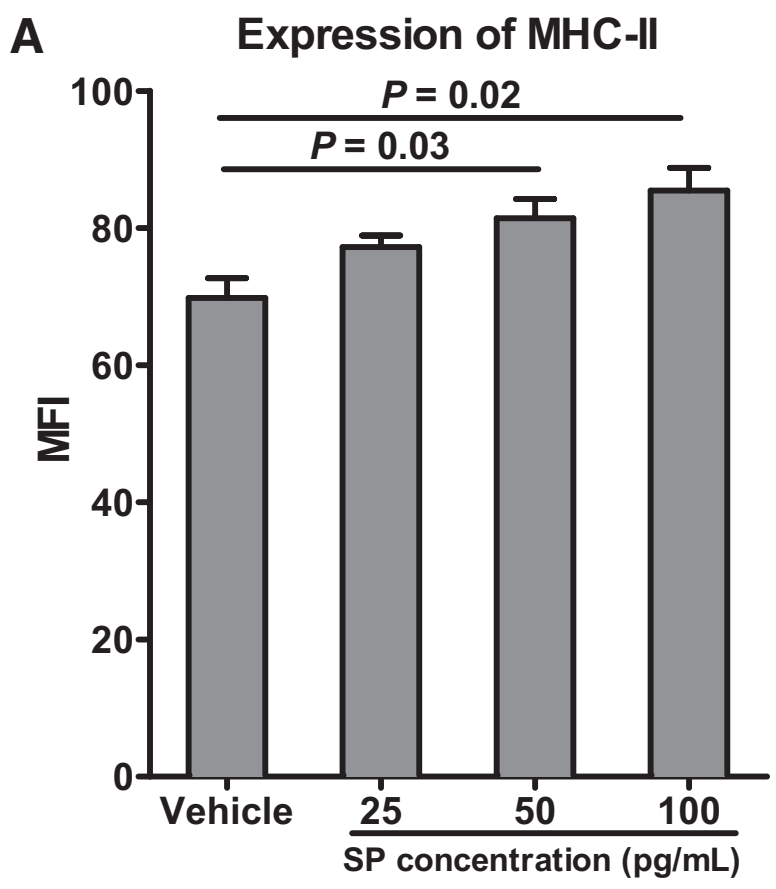

B Expression of MHC-II

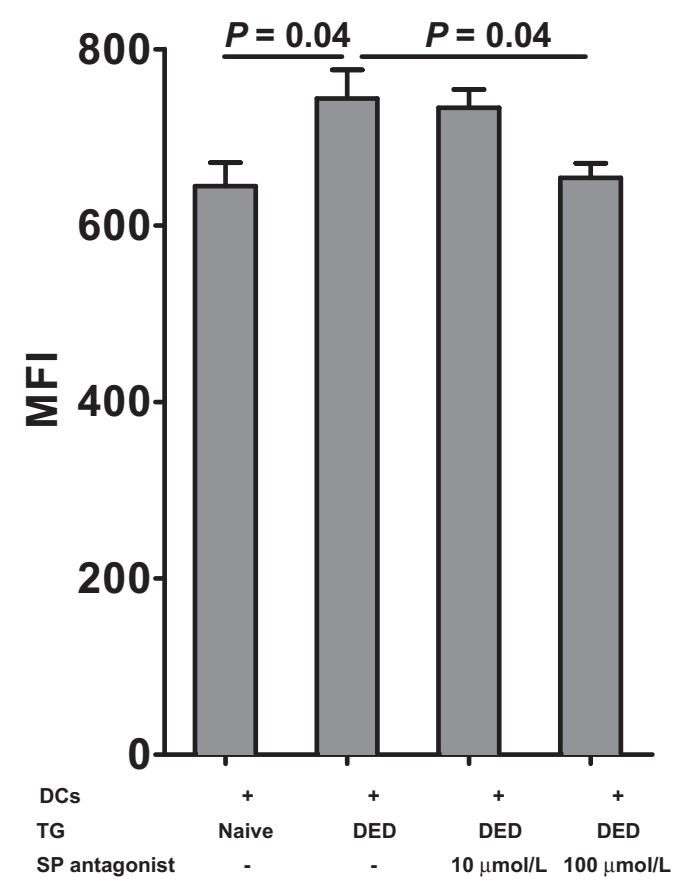

Figure 2 Substance $\mathrm{P}(\mathrm{SP})$ derived from trigeminal ganglion promotes bone marrow-derived dendritic cell (BMDC) maturation. A: BMDCs were cultured in the presence of different doses of substance $P(25,50$, and 100 $\mathrm{pg} / \mathrm{mL}$ ) for 18 hours. Maturation of BMDCs was evaluated by assessing the expression [mean fluorescence intensity (MFI)] of major histocompatibility complex class II (MHC-II) using flow cytometry. B: Trigeminal ganglia (TGs) harvested from either dry eye disease (DED) or control mice were cultured for 3 days and were subsequently co-cultured with BMDCs for 24 hours. To block the effect of TG-derived SP on BMDCs, neurokinin-1 receptor antagonist spantide (10 and $100 \mu \mathrm{mol} / \mathrm{L}$ ) was added to the co-culture system. Expression of MHC-II by CD11C ${ }^{+}$BMDCs was assessed in different groups using flow cytometry. Data are expressed as means $\pm \operatorname{SEM}(\mathbf{A}$ and $\mathbf{B}) . n=2$ independent experiments (A and $\mathbf{B}$ ). mice $(743 \pm 32$ versus $644 \pm 26 ; P=0.04)$ (Figure 2B). Finally, blockade of SP with spantide at the concentration of $100 \mu \mathrm{mol} / \mathrm{L}$ significantly abrogated the effect of DED TG on BMDC maturation (Figure 2B), implicating SP derived from TG neurons in the up-regulated expression of MHC-II by BMDCs.

\section{NK1R Antagonists Ameliorate DED and Inhibit} Antigen-Presenting Cell Maturation in the Cornea and Draining Lymph Nodes

Having shown the in vitro effect of NK1R antagonists in abrogating SP-induced APC maturation, the effect of topical blockade of SP receptor on the clinical signs of DED was examined next. After the induction of DED for 4 days, mice received topical application of either NK1R antagonist CP99,994 or L-733,060 or PBS control three times per day until day 14 after DED induction. Compared with untreated and PBS-treated controls, topical application of either NK1R antagonist CP-99,994 or L-733,060 significantly reduced corneal fluorescein staining scores and DED severity at day 7 , 10, and 14 after DED induction (Figure 3A). To confirm the effect of topical blockade of SP on APC maturation in DED mice, the frequencies of $\mathrm{MHC}-\mathrm{II}^{\mathrm{hi}} \mathrm{CD} 11 \mathrm{~b}^{+}$cells were examined in the cornea and DLNs using flow cytometry (Figure 3, B and C). The cornea of untreated and PBS-treated DED mice showed a significant increase in the frequencies of MHC-II ${ }^{\text {hi }} \mathrm{CD} 11 \mathrm{~b}^{+}$cells compared with that of naïve mice. However, the frequencies of MHC-II ${ }^{\mathrm{hi}} \mathrm{CD} 11 \mathrm{~b}^{+}$cells in the cornea were significantly lower in both CP-99,994- and L-733,060-treated mice (Figure 3B). Similarly, the DLNs of CP-99,994- and L-733,060-treated mice showed a significant decrease in the frequencies of mature $\mathrm{MHC}-\mathrm{II}^{\mathrm{hi}} \mathrm{CD} 11 \mathrm{~b}^{+}$ cells (Figure 3, C and D) as well as the level of MHC-II expression by $\mathrm{CD}_{11} \mathrm{~b}^{+}$cells (Figure 3E) compared with that of PBS-treated mice.

NK1R Antagonists Suppress Activation of Th17 Cells in DLNs and Their Infiltration in the Conjunctivae

The effect of topical blockade of SP signaling on Th17 cell activation was next analyzed in DLNs and conjunctivae. The DLNs of untreated and PBS-treated mice showed an increase (1- to 1.5 -fold) in the frequencies of Th17 cells compared with that of naïve mice. However, topical application of either NK1R antagonist significantly decreased the frequencies of Th17 cells in the DLNs (Figure 4, A and B). A similar decreasing trend in the frequencies of Th17 cells in the conjunctivae of NK1R antagonist-treated mice was observed (Figure 4, C and D). In addition, the real-time PCR data demonstrated a significant reduction in mRNA expression levels of inflammatory cytokine ILI7 in conjunctivae of NK1R antagonist-treated mice (Figure 4E), further confirming the effect of blocking SP signaling on suppressing the Th17 immune response in DED mice. 
A

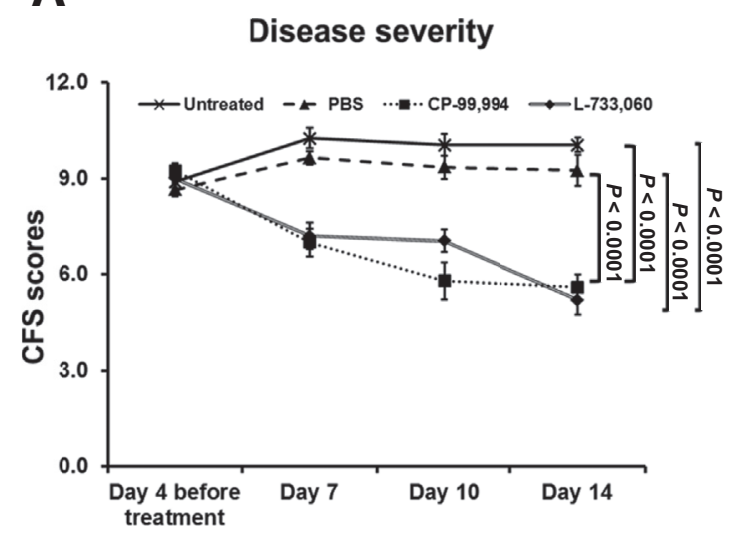

D

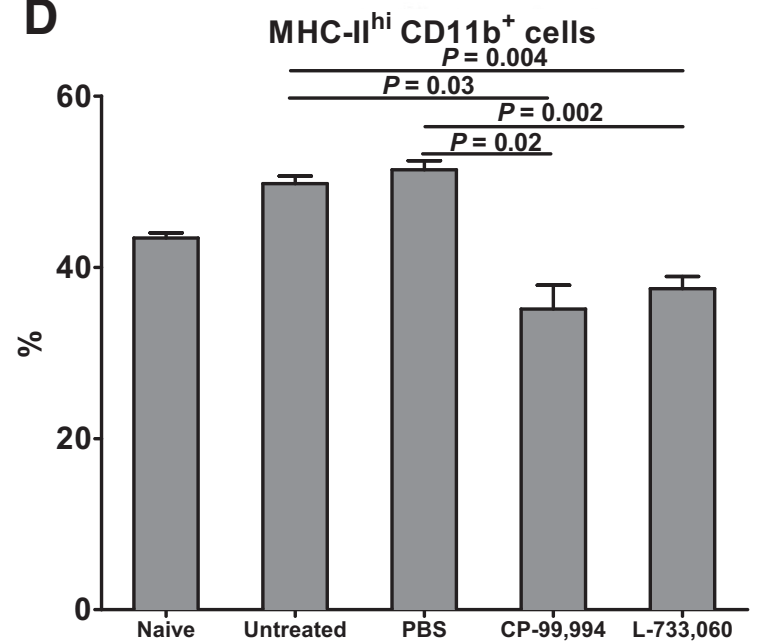

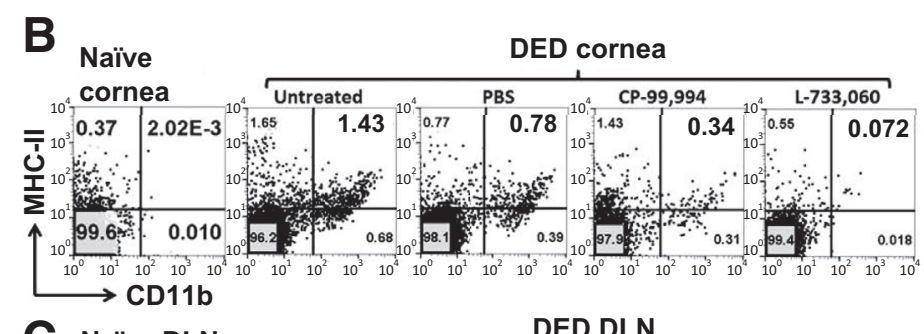



E

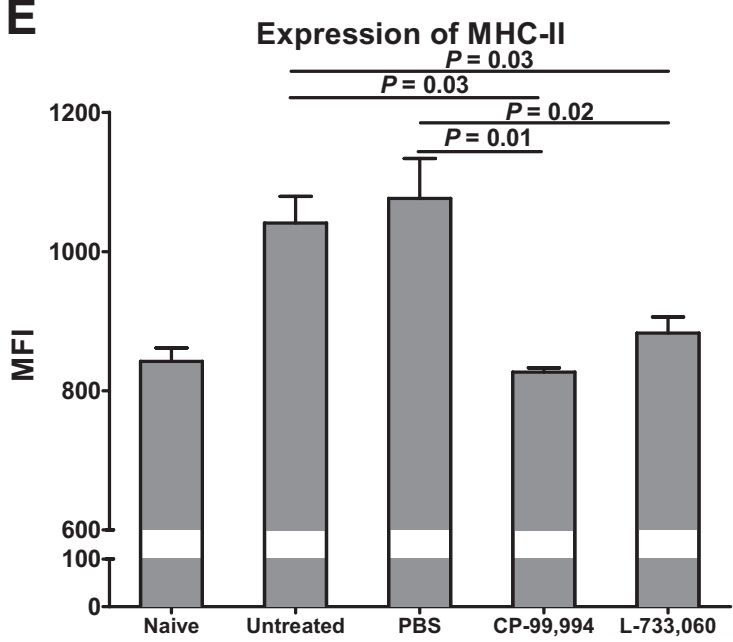

Figure 3 Topical blockade of neurokinin-1 receptor (NK1R) inhibits antigen-presenting cell maturation and ameliorates dry eye disease (DED). After induction of DED for 4 days, mice were topically treated with NK1R antagonist, CP-99,994 or L-733,060 $(1 \mu \mathrm{g} / \mu \mathrm{L})$, or phosphate-buffered saline (PBS; as the control) three times a day until day 14 after DED induction. Untreated DED mice served as controls. A: Corneal fluorescein staining (CFS) was performed on days $4,7,10$, and 14 to assess disease severity. B-E: Frequencies of mature major histocompatibility complex class II (MHC-II) $)^{\text {hi }}$ CD11 b $^{+}$antigen-presenting cells (APCs) in the cornea (B) and draining lymph nodes (DLNs; C and $\mathbf{D}$ ) and expression of MHC-II by APCs in the draining lymph nodes (E) on day 14 were assessed in different groups using flow cytometry. Data are expressed as means \pm SEM (D and $\mathbf{E}) . n=2$ independent experiments (D and $\mathbf{E})$. MFI, mean fluorescence intensity.

\section{Discussion}

Neurogenic inflammation has long been implicated as a potential mechanism involved in the development and chronicity of DED. ${ }^{31,32}$ However, the precise role of neuromodulators, such as SP, in the pathogenesis of DED has not been elucidated. ${ }^{33}$ Herein, it was demonstrated that TG is the major source of SP in the course of DED. SP derived from TG enhanced the expression of MHC-II by BMDCs (a critical mechanism linked to DC antigen-presenting function), and this effect was abrogated by blockade of SP signaling using NK1R antagonist spantide. Finally, using a well-established murine model of DED, it was shown that topical treatment of DED mice with NK1R antagonists CP99,994 and L-733,060 suppresses APC acquisition of MHCII, reduces Th17 cell infiltration and activity, and ameliorates DED severity.
The cornea is the most richly innervated tissue in the body, which receives dense sensory nerve fibers from the ophthalmic branch of the trigeminal nerve. A dense network of SP-expressing fibers innervates the basal area of the corneal epithelium, with terminal branches that penetrate the more superficial layers. SP is known as a key molecule in the cross communications between neural and immune systems. Although neuronal cells serve as the main source of SP, endogenous expression of SP has also been demonstrated in immune cells, keratocytes, and epithelial cells. ${ }^{11,34}$ Thus far, few studies have studied the alterations in SP levels in DED. In a study by Chao et $\mathrm{al}^{35}$ on patients who underwent laser vision correction surgery, the authors showed a positive correlation between SP levels in the tear and dry eye symptoms 1 year after surgery. These results demonstrate significantly higher baseline expression levels of SP in the TG neurons compared with the cornea, both of 

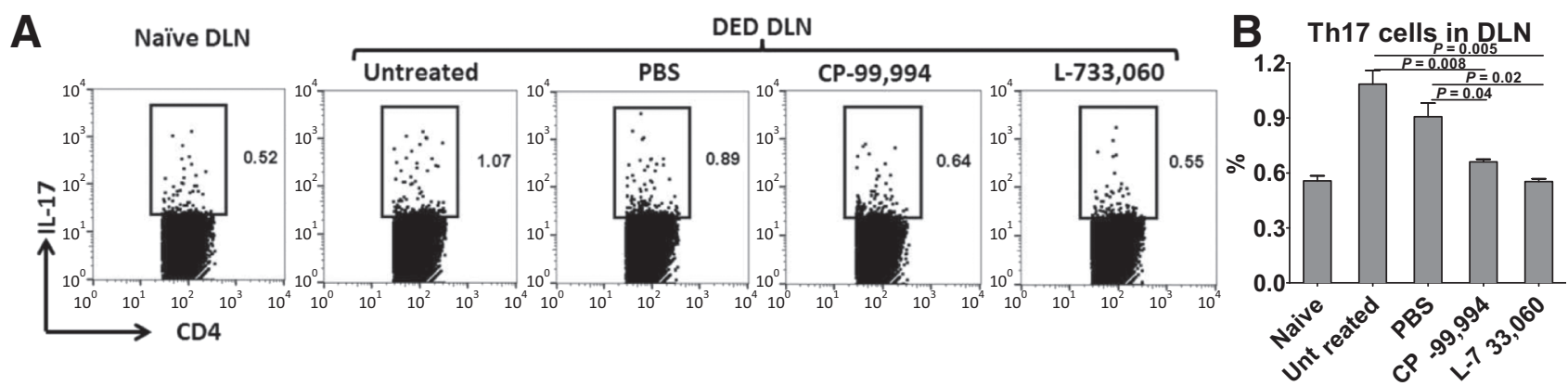

C

Naïve CONJ

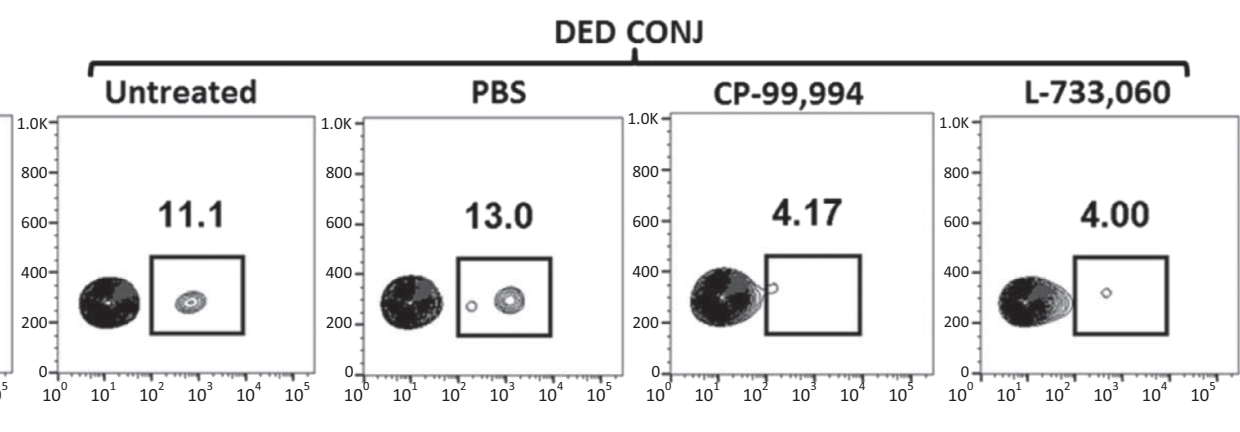

D
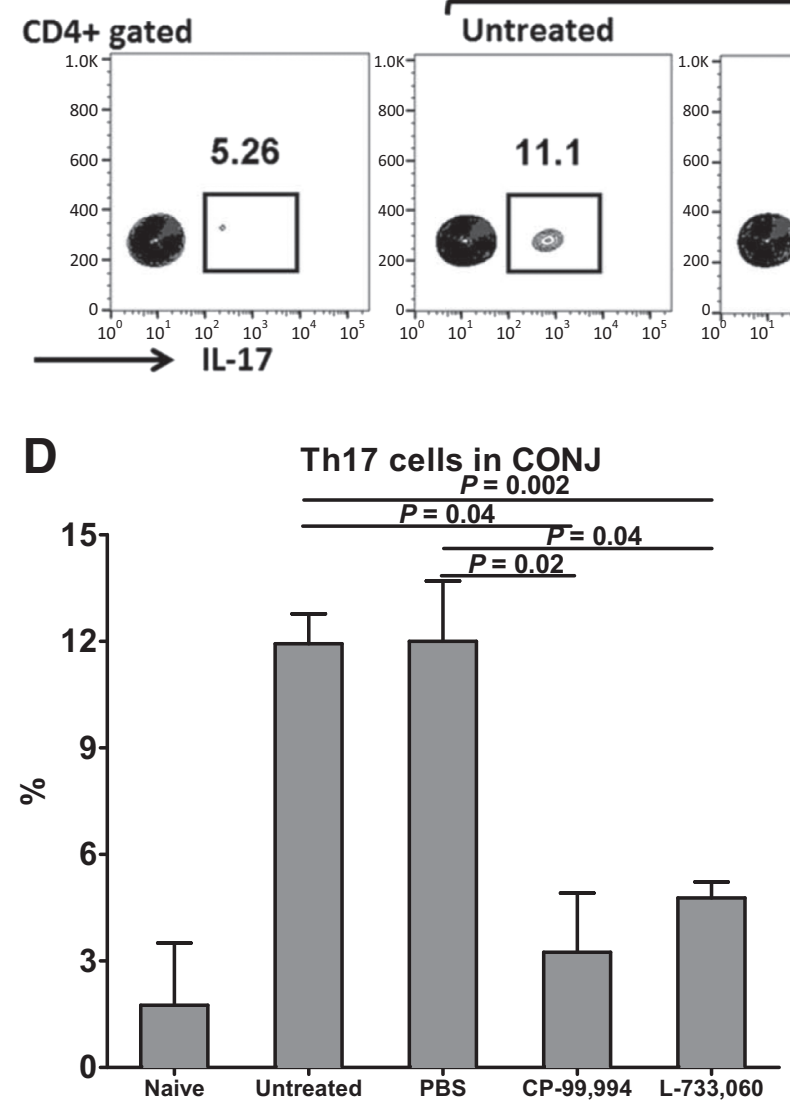

E mRNA expression of IL-17 in CONJ

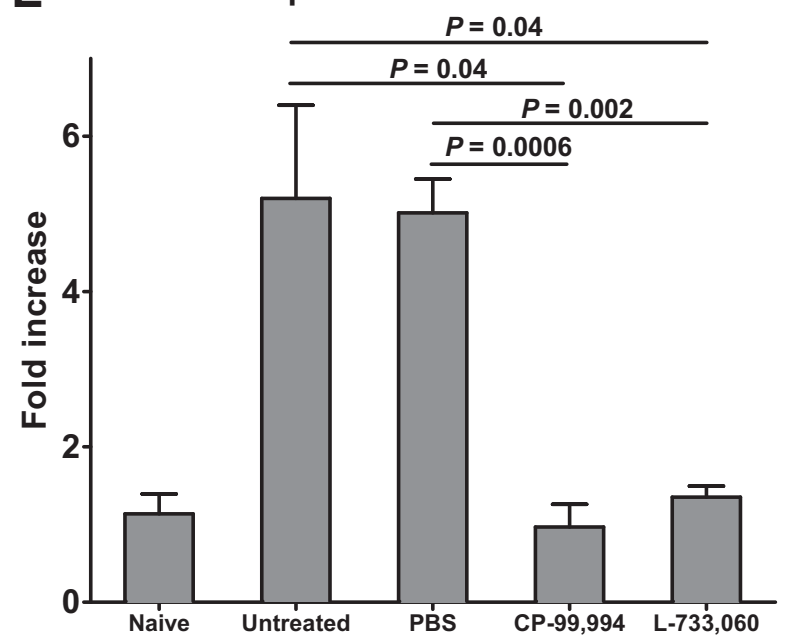

Figure 4 Topical blockade of neurokinin-1 receptor (NK1R) suppresses activation of type 17 helper T (Th17) cells in draining lymph nodes (DLNs) and their infiltration in the conjunctivae (CONJ). After induction of dry eye disease (DED) for 4 days, mice received topical treatment with NK1R antagonist, CP-99,994 or L-733,060 $(1 \mu \mathrm{g} / \mu \mathrm{L})$, or phosphate-buffered saline (PBS; as the control) three times a day until day 14 after DED induction. Untreated DED mice served as the control. A and B: Frequencies of CD4 ${ }^{+}$IL-17 ${ }^{+}$Th17 cells in the DLN of naïve, DED mice treated with CP-99,994, L-733,060, or PBS and untreated DED mice were evaluated on day 14 after DED induction using flow cytometry. C and D: Frequencies of Th17 cells in the conjunctivae of naïve, NK1R antagonist-treated and untreated DED mice. E: mRNA expression levels of IL17 in the conjunctivae of different groups were assessed on day 14 using flow cytometry and real-time PCR. Data are expressed as means \pm SEM (B, D, and $\mathbf{E}) . n=2$ independent experiments $(\mathbf{B}, \mathbf{D}$, and $\mathbf{E})$.

which are up-regulated in response to desiccating stress. The fact that neuronal SP mRNA levels in these experiments increase at a later time point after induction of DED suggests that the early expression of SP at the protein level may be due to the preformed protein in the corneal epithelium.

APCs, including DCs, play a pivotal role in the regulation of immune responses at the interface of innate and adaptive immunity. ${ }^{36}$ A heterogeneous population of tissue-resident DCs has been described in the corneal epithelium and stroma. $^{37}$ Under inflammatory conditions, these DCs undergo the maturation process and acquire antigenpresenting capacity to stimulate $\mathrm{T}$ lymphocyte-dependent responses. ${ }^{38}$ The presence of mature MHC-II ${ }^{+}$DCs has been observed in the course of a wide variety of immunoinflammatory conditions, including dry eye disease. ${ }^{39}$ The homing of mature APCs from the ocular surface to the DLN is a critical step in the early immunopathogenesis of DED. ${ }^{28,29}$ APC-mediated priming of effector T cells has been proposed as a potential source of autoimmunity in DED. ${ }^{28,29}$ Interestingly, several studies have reported APCs 
to be located in close proximity of SP-immunoreactive nerves in diverse organs ${ }^{40-42}$ and have suggested SP to modify the function and migration of DCs. ${ }^{43-48}$ The results of the TG-BMDC co-culture demonstrate that TG-derived SP enhances the expression of MHC class II by DCs, thus enhancing their antigen-presenting capacity. Antagonizing NK1R in the co-culture interestingly abrogates SP-induced DC maturation, further demonstrating the role of SP signaling in inducing APC activation.

Previous studies have assessed the application of NK1R antagonists in animal models of ocular surface disorders, such as pseudomonas keratitis, ${ }^{18}$ herpetic stromal keratitis, ${ }^{19}$ and corneal neovascularization. ${ }^{20}$ It has been confirmed that native and cultured corneal epithelial cells express NK1R. ${ }^{49,50}$ However, the efficacy of NK1R antagonists in animal models of DED has not as of yet been described. To evaluate their efficacy in the setting of DED, two different, highly specific, and potent NK1R antagonists (CP-99,994, and L-733,060) were tested. These two inhibitors were chosen as they are readily soluble in aqueous solution. Topical application of either CP-99,994 or L-733,060 suppressed APC maturation and Th17 activity, resulting in amelioration of corneal epitheliopathy. The application of CP-99,994 has not been previously reported in ocular diseases. However, SP is a pleiotropic molecule with myriad functions, including in physiological homeostasis of the ocular surface. Relatedly, Yang et $\mathrm{al}^{51}$ showed that topical application of SP promotes corneal epithelial wound healing in diabetic mice and subconjunctival injection of L-733,060 significantly inhibits the protective role of SP on epithelial healing. Despite the potential delay in wound healing, strong evidence supports the therapeutic implications of NK1R antagonists in both clinical ${ }^{52}$ and experimental ${ }^{18}$ ocular inflammation. As such, similar to corticosteroids, NK1R antagonists may be seen as double-edged swords whose efficacy in modulating pathobiology has to be weighed carefully against their potential to unfavorably influence wound healing.

In summary, consistent with previous reports, ${ }^{53,54}$ these data on the increased frequencies of MHC-II ${ }^{\text {hi }} \mathrm{CD} 11 \mathrm{~b}^{+}$ APCs in the cornea and DLNs after the induction of DED demonstrate the effect of desiccating stress in enhancing the maturation of resident corneal APCs and their migration toward the DLNs. Treatment of DED mice with NK1R antagonists significantly decreases the frequencies of mature APCs in the cornea and DLNs, where APCs prime naïve T cells to differentiate into IL-17-secreting Th17 cells. The observed suppressive effect of NK1R antagonists on APC maturation prompted us to assess their efficacy in inhibiting Th17 cell activation. As hypothesized, a substantial decrease in the frequencies of Th17 cells was observed in both the DLNs and the conjunctivae of NK1R antagonist-treated mice, which was associated with a marked reduction in IL17 mRNA levels.

Antagonizing NK1R-mediated signaling may be an effective strategy in suppressing Th17-mediated ocular surface disease. Clearly, additional studies are warranted to systematically evaluate the optimal timing and dosing of NK1R antagonist(s) for maximally suppressing DEDrelated ocular surface inflammation, while minimizing the potential for untoward adverse effects.

\section{Acknowledgment}

M.Y. thanks the China Scholarship Council for individual training fellowship support.

\section{References}

1. Schaumberg DA, Dana R, Buring JE, Sullivan DA: Prevalence of dry eye disease among US men: estimates from the Physicians' Health Studies. Arch Ophthalmol 2009, 127:763-768

2. Chen Y, Chauhan SK, Lee HS, Saban DR, Dana R: Chronic dry eye disease is principally mediated by effector memory Th17 cells. Mucosal Immunol 2014, 7:38-45

3. Chauhan SK, El Annan J, Ecoiffier T, Goyal S, Zhang Q, Saban DR, Dana R: Autoimmunity in dry eye is due to resistance of Th17 to Treg suppression. J Immunol 2009, 182:1247-1252

4. Vickers LA, Gupta PK: The future of dry eye treatment: a glance into the therapeutic pipeline. Ophthalmol Ther 2015, 4:69-78

5. Pflugfelder SC, Corrales RM, de Paiva CS: T helper cytokines in dry eye disease. Exp Eye Res 2013, 117:118-125

6. De Paiva CS, Chotikavanich S, Pangelinan SB, Pitcher JD 3rd, Fang B, Zheng X, Ma P, Farley WJ, Siemasko KF, Niederkorn JY, Stern ME, Li DQ, Pflugfelder SC: IL-17 disrupts corneal barrier following desiccating stress. Mucosal Immunol 2009, 2:243-253

7. Chauhan SK, Dana R: Role of Th17 cells in the immunopathogenesis of dry eye disease. Mucosal Immunol 2009, 2:375-376

8. Snijdelaar DG, Dirksen R, Slappendel R, Crul BJ: Substance P. Eur J Pain 2000, 4:121-135

9. Bost KL: Tachykinin-modulated anti-viral responses. Front Biosci 2004, 9:1994-1998

10. Nelson DA, Bost KL: Non-neuronal mammalian tachykinin expression. Front Biosci 2004, 9:2166-2176

11. O'Connor TM, O'Connell J, O'Brien DI, Goode T, Bredin CP, Shanahan F: The role of substance $\mathrm{P}$ in inflammatory disease. J Cell Physiol 2004, 201:167-180

12. Dunnick CA, Gibran NS, Heimbach DM: Substance P has a role in neurogenic mediation of human burn wound healing. J Burn Care Rehabil 1996, 17:390-396

13. Lai X, Wang Z, Wei L, Wang L: Effect of substance P released from peripheral nerve ending on endogenous expression of epidermal growth factor and its receptor in wound healing. Chin J Traumatol 2002, 5:176-179

14. Nakamura M, Ofuji K, Chikama T, Nishida T: Combined effects of substance $\mathrm{P}$ and insulin-like growth factor-1 on corneal epithelial wound closure of rabbit in vivo. Curr Eye Res 1997, 16:275-278

15. Tuluc F, Lai JP, Kilpatrick LE, Evans DL, Douglas SD: Neurokinin 1 receptor isoforms and the control of innate immunity. Trends Immunol 2009, 30:271-276

16. Hong Y, Lee J, Lee E, Kwon Y, Lee E, Ahn W, Jiang M, Kim J, Son Y: A new role of substance $\mathrm{P}$ as an injury-inducible messenger for mobilization of CD29+ stromal-like cells. Nat Med 2005, 15: 425-435

17. Chao C, Stapleton F, Zhou X, Chen S, Zhou S, Golebiowski B: Structural and functional changes in corneal innervation after laser in situ keratomileusis and their relationship with dry eye. Graefes Arch Clin Exp Ophthalmol 2015, 253:2029-2039

18. Hazlett LD, McClellan SA, Barrett RP, Liu J, Zhang Y, Lighvani S: Spantide I decreases type I cytokines, enhances IL-10, and reduces 
corneal perforation in susceptible mice after Pseudomonas aeruginosa infection. Invest Ophthalmol Vis Sci 2007, 48:797-807

19. Twardy BS, Channappanavar R, Suvas S: Substance P in the corneal stroma regulates the severity of herpetic stromal keratitis lesions. Invest Ophthalmol Vis Sci 2011, 52:8604-8613

20. Bignami F, Giacomini C, Lorusso A, Aramini A, Rama P, Ferrari G: NK1 receptor antagonists as a new treatment for corneal neovascularization. Invest Ophthalmol Vis Sci 2014, 55:6783-6794

21. Cunin P, Caillon A, Corvaisier M, Garo E, Scotet M, Blanchard S, Delneste Y, Jeannin P: The tachykinins substance P and hemokinin-1 favor the generation of human memory Th17 cells by inducing IL1beta, IL-23, and TNF-like 1A expression by monocytes. J Immunol 2011, 186:4175-4182

22. Takashima A: Harnessing DCs by substance P. Blood 2013, 121: $2815-2816$

23. Mashaghi A, Marmalidou A, Tehrani M, Grace PM, Pothoulakis C, Dana R: Neuropeptide substance P and the immune response. Cell Mol Life Sci 2016, 73:4249-4264

24. Chen Y, Chauhan SK, Tan X, Dana R: Interleukin-7 and -15 maintain pathogenic memory Th17 cells in autoimmunity. J Autoimmun 2017 , 77:96-103

25. Chen Y, Chauhan SK, Shao C, Omoto M, Inomata T, Dana R: IFNgamma-expressing Th17 cells are required for development of severe ocular surface autoimmunity. J Immunol 2017, 199:1163-1169

26. Bertke AS, Swanson SM, Chen J, Imai Y, Kinchington PR, Margolis TP: A5-positive primary sensory neurons are nonpermissive for productive infection with herpes simplex virus 1 in vitro. J Virol 2011, 85:6669-6677

27. Malin SA, Davis BM, Molliver DC: Production of dissociated sensory neuron cultures and considerations for their use in studying neuronal function and plasticity. Nat Protoc 2007, 2:152-160

28. Barabino S, Chen Y, Chauhan S, Dana R: Ocular surface immunity: homeostatic mechanisms and their disruption in dry eye disease. Prog Retin Eye Res 2012, 31:271-285

29. Stevenson W, Chauhan SK, Dana R: Dry eye disease: an immunemediated ocular surface disorder. Arch Ophthalmol 2012, 130:90-100

30. Hamrah P, Liu Y, Zhang Q, Dana MR: The corneal stroma is endowed with a significant number of resident dendritic cells. Invest Ophthalmol Vis Sci 2003, 44:581-589

31. Beuerman RW, Stern ME: Neurogenic inflammation: a first line of defense for the ocular surface. Ocul Surf 2005, 3:S203-S206

32. Stern ME, Gao J, Siemasko KF, Beuerman RW, Pflugfelder SC: The role of the lacrimal functional unit in the pathophysiology of dry eye. Exp Eye Res 2004, 78:409-416

33. Sabatino F, Di Zazzo A, De Simone L, Bonini S: The intriguing role of neuropeptides at the ocular surface. Ocul Surf 2017, 15:2-14

34. Watanabe M, Nakayasu K, Iwatsu M, Kanai A: Endogenous substance P in corneal epithelial cells and keratocytes. Jpn J Ophthalmol 2002, 46:616-620

35. Chao C, Golebiowski B, Zhao X, Chen S, Zhou S, Stapleton F: Longterm effects of LASIK on corneal innervation and tear neuropeptides and the associations with dry eye. J Refract Surg 2016, 32:518-524

36. Fransen JH, van der Vlag J, Ruben J, Adema GJ, Berden JH, Hilbrands LB: The role of dendritic cells in the pathogenesis of systemic lupus erythematosus. Arthritis Res Ther 2010, 12:207

37. Hamrah P, Huq SO, Liu Y, Zhang Q, Dana MR: Corneal immunity is mediated by heterogeneous population of antigen-presenting cells. J Leukoc Biol 2003, 74:172-178

38. Liu YJ, Kanzler H, Soumelis V, Gilliet M: Dendritic cell lineage, plasticity and cross-regulation. Nat Immunol 2001, 2:585-589

39. Catry L, Van den Oord J, Foets B, Missotten L: Morphologic and immunophenotypic heterogeneity of corneal dendritic cells. Graefes Arch Clin Exp Ophthalmol 1991, 229:182-185
40. Okiji T, Jontell M, Belichenko P, Dahlgren U, Bergenholtz G, Dahlstrom A: Structural and functional association between substance $\mathrm{P}$ - and calcitonin gene-related peptide-immunoreactive nerves and accessory cells in the rat dental pulp. J Dent Res 1997, 76:1818-1824

41. Kradin R, MacLean J, Duckett S, Schneeberger EE, Waeber C, Pinto C: Pulmonary response to inhaled antigen: neuroimmune interactions promote the recruitment of dendritic cells to the lung and the cellular immune response to inhaled antigen. Am J Pathol 1997, 150:1735-1743

42. Markus AM, Kockerling F, Neuhuber WL: Close anatomical relationships between nerve fibers and MHC class II-expressing dendritic cells in the rat liver and extrahepatic bile duct. Histochem Cell Biol 1998, 109:409-415

43. Arck PC, Handjiski B, Peters EM, Peter AS, Hagen E, Fischer A, Klapp BF, Paus R: Stress inhibits hair growth in mice by induction of premature catagen development and deleterious perifollicular inflammatory events via neuropeptide substance P-dependent pathways. Am J Pathol 2003, 162:803-814

44. Janelsins BM, Sumpter TL, Tkacheva OA, Rojas-Canales DM, Erdos G, Mathers AR, Shufesky WJ, Storkus WJ, Falo LD Jr, Morelli AE, Larregina AT: Neurokinin-1 receptor agonists bias therapeutic dendritic cells to induce type 1 immunity by licensing host dendritic cells to produce IL-12. Blood 2013, 121: 2923-2933

45. Joachim RA, Handjiski B, Blois SM, Hagen E, Paus R, Arck PC: Stress-induced neurogenic inflammation in murine skin skews dendritic cells towards maturation and migration: key role of intercellular adhesion molecule-1/leukocyte function-associated antigen interactions. Am J Pathol 2008, 173:1379-1388

46. O'Connell PJ, Pingle SC, Ahern GP: Dendritic cells do not transduce inflammatory stimuli via the capsaicin receptor TRPV1. FEBS Lett 2005, 579:5135-5139

47. Divito SJ, Morelli AE, Larregina AT: Role of neurokinin-1 receptor in the initiation and maintenance of skin chronic inflammatory diseases. Immunol Res 2011, 50:195-201

48. Pavlovic S, Liezmann C, Blois SM, Joachim R, Kruse J, Romani N, Klapp BF, Peters EM: Substance P is a key mediator of stress-induced protection from allergic sensitization via modified antigen presentation. J Immunol 2011, 186:848-855

49. Denis P, Fardin V, Nordmann JP, Elena PP, Laroche L, Saraux H, Rostene W: Localization and characterization of substance P binding sites in rat and rabbit eyes. Invest Ophthalmol Vis Sci 1991, 32: $1894-1902$

50. Nakamura M, Ofuji K, Chikama T, Nishida T: The NK1 receptor and its participation in the synergistic enhancement of corneal epithelial migration by substance $\mathrm{P}$ and insulin-like growth factor- 1 . Br J Pharmacol 1997, 120:547-552

51. Yang L, Di G, Qi X, Qu M, Wang Y, Duan H, Danielson P, Xie L, Zhou Q: Substance P promotes diabetic corneal epithelial wound healing through molecular mechanisms mediated via the neurokinin-1 receptor. Diabetes 2014, 63:4262-4274

52. Lucas K, Karamichos D, Mathew R, Zieske JD, Stein-Streilein J: Retinal laser burn-induced neuropathy leads to substance P-dependent loss of ocular immune privilege. J Immunol 2012, 189: $1237-1242$

53. Goyal S, Chauhan SK, El Annan J, Nallasamy N, Zhang Q, Dana R: Evidence of corneal lymphangiogenesis in dry eye disease: a potential link to adaptive immunity? Arch Ophthalmol 2010, 128: 819-824

54. Kodati S, Chauhan SK, Chen Y, Dohlman TH, Karimian P, Saban D, Dana R: CCR7 is critical for the induction and maintenance of Th17 immunity in dry eye disease. Invest Ophthalmol Vis Sci 2014, 55: 5871-5877 